\title{
THE SPECTRAL MEASURE AND HILBERT TRANSFORM OF A MEASURE-PRESERVING TRANSFORMATION
}

\author{
JAMES CAMPBELL AND KARL PETERSEN
}

\begin{abstract}
V. F. Gaposhkin gave a condition on the spectral measure of a normal contraction on $L^{2}$ sufficient to imply that the operator satisfies the pointwise ergodic theorem. We prove that unitary operators which come from measure-preserving transformations satisfy a stronger version of this condition. This follows from the fact that the rotated ergodic Hilbert transform is a continuous function of its parameter. The maximal inequality on which the proof depends follows from an analytic inequality related to the Carleson-Hunt Theorem on the a.e. convergence of Fourier series.
\end{abstract}

There is a large body of work on the question of when a given operator on an $L^{p}$ space satisfies the pointwise ergodic theorem, that is, when the Cesàro means of powers of the operator applied to an element of $L^{p}$ converge a.e. (See the book by Krengel [1985] and the article by Duncan [1977] for surveys.) Gaposhkin [1981] gave a necessary and sufficient condition involving the spectral measure of the operator for the case when $p=2$ and the operator in question is a normal contraction. In this paper we develop a connection (Proposition 1) between this condition and the ergodic Hilbert transform. This connection allows us to prove directly that operators induced by measure-preserving transformations satisfy a strengthened version of Gaposhkin's condition (Theorem 1). The fundamental result is a form of continuity of the rotated ergodic Hilbert transform (Theorem 2), the proof of which depends on a new kind of maximal inequality involving a supremum over a parameter (Lemma 1). We reduce the proof of this inequality to an analytic maximal inequality (Lemma 2), which is proved from the Carleson-Hunt estimate on maxima of partial sums of Fourier series.

We thank the many people with whom we discussed this work for their valuable contributions, especially $M$. Taylor, who provided the key ideas for the proof of Lemma 2, and M. Keane, S. Chanillo, and T. A. Gillespie.

Let $(X, \mathscr{B}, \mu)$ denote a measure space, and let $T$ be a normal contraction on $L^{2}(X, \mathscr{B}, \mu)$. Let $E_{T}$ denote the spectral measure for $T$, supported on the closed unit disc in the complex plane, and for each $n=1,2, \ldots$ let

Received by the editors March 15, 1987.

1980 Mathematics Subject Classification (1985 Revision). Primary 28D05, 47A60, 47 A35.

Key words and phrases. Spectral measure, ergodic Hilbert transform, maximal inequality.

Research supported in part by NSF grants DMS-8400730 and DMS-8620132. 
$V_{n}=\left\{z \in \mathbf{C}: 0<|1-z|<2^{-n}\right\}$. In $1981 \mathrm{~V}$. F. Gaposhkin proved that for a given $f \in L^{2}$ the pointwise ergodic theorem holds for $T$ and $f$, i.e.,

$$
\lim _{n \rightarrow \infty} \frac{1}{n} \sum_{k=0}^{n-1} T^{k} f(x) \text { exists a.e. }
$$

if and only if

$$
\lim _{n \rightarrow \infty}\left[E_{T}\left(V_{n}\right) f\right](x)=0 \text { a.e. }
$$

Moreover, he showed that the sequence $2^{-n}$ used in the definition of $V_{n}$ may be replaced by any sequence $q_{n}$ converging monotonically to 0 and satisfying $Q \geq q_{n} / q_{n+1} \geq q>1$ for every $n$.

Gaposhkin observed that since the pointwise ergodic theorem is known to hold for all $f \in L^{2}$, if $T$ is an invertible measure-preserving (m.p.t.) on $X$, it would be interesting to verify (2) directly for operators induced by such transformations. (If the measure-preserving transformation is not invertible, then the operator that is induces is not normal.) Our main result shows that for m.p.t.'s a strengthened version of (2) actually holds:

Theorem 1. Let $T$ be an invertible m.p.t. on a measure space $(X, \mathscr{B}, \mu)$ with associated spectral representation

$$
T=\int_{-\pi}^{\pi} e^{i \lambda} d E(\lambda)
$$

as an operator on $L^{2}$. If $\left\{\varepsilon_{k}\right\}$ is any nonnegative sequence which tends to 0 as $k \rightarrow \infty$, then

$$
\lim _{k \rightarrow \infty}\left[E\left(-\varepsilon_{k}, 0\right) f\right](x)=0 \text { a.e. for all } f \in L^{2} \text {. }
$$

We prove this theorem by formulating a condition equivalent to (4) and then verifying this new condition. The condition we will formulate depends upon the ergodic Hilbert transform (e.H.t.). Recall that for $f \in L^{2}$ the e.H.t. of $f$ (induced by $T$ ) is the a.e. limit

$$
\lim _{n \rightarrow \infty} \frac{1}{\pi} \sum_{k=-n}^{n} \frac{f\left(T^{k} x\right)}{k}=\mathrm{P} . \mathrm{V} \cdot \frac{1}{\pi} \sum_{k=-\infty}^{\infty} \frac{f\left(T^{k} x\right)}{k}=H_{T} f(x),
$$

where' denotes omission of the term for which $k=0$. Usually $T$ is understood and we just write $H f$. The map which sends $f$ to $H f$ is a bounded operator on $L^{2}$ (see Cotlar [1955]). If $T$ has the representation (3), then $H$ may be represented via the spectral integral

$$
H=i \int_{-\pi}^{\pi} \eta(\lambda) d E(\lambda)
$$

where $\eta(\lambda)$ is the odd function on $[-\pi, \pi]$ whose value for $\lambda \in(0, \pi]$ is $(\pi-\lambda) / \pi$ and $\eta(0)=0$. (See Campbell [1986].) 
By considering a simple product transformation it is easy to see that for fixed $\varepsilon$ in $[-\pi, \pi]$, the rotated e.H.t., $H_{\varepsilon} f$, defined by

$$
H_{\varepsilon} f(x)=\text { P.V. } \frac{1}{\pi} \sum_{k=-\infty}^{\infty} \frac{e^{i k \varepsilon} f\left(T^{k} x\right)}{k}
$$

exists a.e. for each $f \in L^{2}$, and the map sending $f$ to $H_{\varepsilon} f$ is a bounded operator on $L^{2}$. We claim that (4) above is equivalent to a form of continuity of the rotated Hilbert transform at $\varepsilon=0$ :

Proposition 1. For $f \in L^{2}, T$, and $E$ as above,

$$
\lim _{k \rightarrow \infty}\left[E\left(-\varepsilon_{k}, 0\right) f\right](x)=0 \text { a.e. }
$$

if and only if

$$
\lim _{k \rightarrow \infty} H_{\varepsilon_{k}} f(x)=H f(x)+i[E\{0\} f](x) \text { a.e. }
$$

Proof. We apply the functional calculus. If $\eta(\lambda)$ is the "representing function" for $H$ as in (6), then $\eta\left(\lambda+\varepsilon_{k}\right)$ is the representing function for $H_{\varepsilon_{k}}$, so that $H_{\varepsilon_{k}}-H$ has the representation

$$
H_{\varepsilon_{k}}-H=i \int_{-\pi}^{\pi}\left[\eta\left(\lambda+\varepsilon_{k}\right)-\eta(\lambda)\right] d E(\lambda)
$$

The difference $\eta\left(\lambda+\varepsilon_{k}\right)-\eta(\lambda)$ may be written as

$$
\left(-\varepsilon_{k} / \pi\right) 1+\chi_{\left\{-\varepsilon_{k}\right\}}(\lambda)+2 \chi_{\left(-\varepsilon_{k}, 0\right)}(\lambda)+\chi_{\{0\}}(\lambda)
$$

where 1 is the function which is identically 1 on $[-\pi, \pi]$ and $\chi_{A}$ is the characteristic function of set $A$. Since $\int_{-\pi}^{\pi} 1 d E(\lambda)=I$, the identity operator on $L^{2}$, we may conclude that (8) holds if and only if

$$
\lim _{k \rightarrow \infty}\left(\frac{-\varepsilon_{k}}{\pi}\right) f(x)+E\left\{-\varepsilon_{k}\right\} f(x)+2 E\left(-\varepsilon_{k}, 0\right) f(x)=0 \text { a.e. }
$$

Clearly, $\lim _{k \rightarrow \infty}-\varepsilon_{k} f(x) / \pi=0$ a.e. for each $f \in L^{2}$. Also,

$$
\lim _{k \rightarrow \infty} E\left(\left\{-\varepsilon_{k}\right\}\right) f(x)=0 \quad \text { a.e. }
$$

for the following reason. If $e^{-i \varepsilon_{k}}$ is an eigenvalue for $T$, then $E\left\{-\varepsilon_{k}\right\}$ is the projection onto the corresponding eigenspace; otherwise it is the 0 projection. No matter what order the eigenvalues are given, as $k \rightarrow \infty$ these projections applied to $f$ give the tail blocks of the eigenfunction expansion for the projection of $f$ into the Kronecker factor. Since these blocks are $L^{2}$ summable, the tail blocks tend to 0 a.e. Thus (8) holds if and only if the third piece of the sum, $2 E\left(\left(-\varepsilon_{k}, 0\right)\right) f(x)$, tends to 0 a.e., as $k$ tends to $\infty$. 
To prove Theorem 1 it is sufficient to prove that (8) holds:

Theorem 2. If $T$ is an invertible m.p.t. on a measure space $(X, \mathscr{B}, \mu)$, and $f \in L^{2}(X, \mathscr{B}, \mu)$, then for any nonnegative sequence $\left\{\varepsilon_{k}\right\}$ tending to 0 as $k \rightarrow \infty$ we have

$$
\lim _{k \rightarrow \infty} H_{\varepsilon_{k}} f(x)=H f(x)+i E\{0\} f(x) \text { a.e. }
$$

Proof. As usual, we prove a.e. convergence for a dense subset of $L^{2}$, and then establish an appropriate maximal inequality (Lemma 1 ). The functional calculus may be used to provide the dense subset. If $f$ is in the range of $E\{0\}$, so that $f(x)=f(T x)=E\{0\} f(x)$ a.e. and $H f(x)=0$ a.e., then $H_{\varepsilon_{k}} f(x)=$ $i \eta\left(\varepsilon_{k}\right) f(x)$ a.e., and $\lim _{k \rightarrow \infty} i \eta\left(\varepsilon_{k}\right) f(x)=i f(x)$ a.e. Suppose now that $\delta>0$ is fixed and $f$ is in the range of $E([-\pi,-\delta) \cup(\delta, \pi])$. Then $E\{0\} f(x)=0$ a.e., and if $0<\varepsilon_{k}<\delta$ we have (see (11)) $\left(H_{\varepsilon_{k}}-H\right) f(x)=i \varepsilon_{k} f(x) / \pi$ a.e., which clearly tends to 0 a.e. as $k \rightarrow \infty$. The union over all positive $\delta$ of such functions, along with those in the range of $E\{0\}$, is dense in $L^{2}$.

To complete the proof of Theorem 2 we prove the following maximal inequality which involves a double supremum:

Lemma 1. For each $f \in L^{2}, \varepsilon>0$ and $n \in \mathbf{N}$, define

$$
H_{n, \varepsilon} f(x)=\frac{1}{\pi} \sum_{k=-n}^{n} \frac{e^{i k \varepsilon} f\left(T^{k} x\right)}{k}
$$

and

$$
H^{*} f(x)=\sup _{n, \varepsilon}\left|H_{n, \varepsilon} f(x)\right| .
$$

Then there exists a constant $C>0$ such that

$$
\mu\left\{x: H^{*} f(x)>\lambda\right\} \leq \frac{C}{\lambda^{2}}\|f\|_{2}^{2} \text { for all } \lambda>0 \text { and } f \in L^{2} .
$$

To prove Lemma 1 we need first to prove Lemma 2, an interesting maximal inequality from harmonic analysis. Then we use Lemma 2 to prove Lemma 3, a sequence version of Lemma 1, which finally transfers to the ergodic setting.

Lemma 2. For $h \in L^{2}[-\pi, \pi]$ (with respect to Lebesgue measure) define a sequence of nonnegative numbers by

$$
I^{*} h(j)=\sup _{\varepsilon>0}\left|\int_{-\varepsilon}^{\varepsilon} h(t) e^{i j t} d t\right|, \quad j \in \mathbf{Z} .
$$

Then there is a constant $C>0$ such that

$$
\left\|I^{*} h(j)\right\|_{l^{2}(\mathbf{Z})} \leq C\|h(t)\|_{L^{2}[-\pi, \pi]} \text { for all } h \in L^{2}[-\pi, \pi] .
$$

Remark. The Carleson-Hunt estimate for the maxima of partial sums of Fourier series of $L^{2}$ functions says that if

$$
S^{*} \widehat{f}(x)=\sup _{n>0}\left|\sum_{k=-n}^{n} \widehat{f}(k) e^{i k x}\right|,
$$


then

$$
\left\|S^{*} \widehat{f}\right\|_{L^{2}[-\pi, \pi]} \leq C\|\widehat{f}\|_{l^{2}(\mathbf{Z})} .
$$

Thus Lemma 2 may be regarded as the Fourier transform of the Carleson-Hunt Theorem. Kenig and Tomas [1980] derived analogues of the Carleson-Hunt result for other dual pairings; the following argument uses their transference of the Carleson-Hunt Theorem to the $(\mathbf{R}, \mathbf{R})$ pairing to prove the version stated in Lemma 2.

Proof of Lemma 2. Fix $h$ and for each complex number $s$ and $\varepsilon>0$ define

$$
G_{\varepsilon}(s)=\left|\int_{-\infty}^{\infty} \chi(t)_{(-\varepsilon, \varepsilon)} h(t) e^{i s t} d t\right|
$$

and

$$
G(s)=\sup _{\varepsilon>0} G_{\varepsilon}(s)
$$

Easy calculations show that

(i) The family $\left\{G_{\varepsilon}: 0 \leq \varepsilon \leq \pi\right\}$ is equicontinuous on the complex plane.

(ii) For each $\varepsilon, G_{\varepsilon}(s)$ is a subharmonic function of $s$.

These statements together are sufficient to imply that $G$ is subharmonic in the plane (see Hörmander [1973, p. 16]). We want to bound the sum of the squares of the values of $G$ at the integers by a constant times the $L^{2}$ norm of $h$. If $D_{j}$ is the disc of radius $1 / 2$ centered at $j$, then by the mean-value property of subharmonic functions and Hölder's inequality we have

$$
G(j)^{2} \leq C\left(\iint_{D_{j}} G(x+i y) d x d y\right)^{2} \leq C \iint_{D_{j}} G(x+i y)^{2} d x d y .
$$

Note that $C$ is independent of $j$ and $h$. The disjoint union of the $D_{j}$ 's is contained in the strip $-1 / 2 \leq y \leq 1 / 2$, and we can estimate the $L^{2}$ norm of $G$ over this strip as follows. For each $y \in[-1 / 2,1 / 2]$, let $G^{y}(x)=G(x+i y)$, so that

$$
G^{y}(x)=\sup _{\varepsilon>0}\left|\int_{-\varepsilon}^{\varepsilon} h(t) e^{-y t} e^{i x t} d t\right| .
$$

For each such $y, F(t)=e^{-y t} h(t)$ is in $L^{2}(\mathbf{R})$, and hence we may apply the result of Kenig-Tomas [1980] to transfer the Carleson-Hunt maximal estimate to this setting:

$$
\left\|G^{y}(x)\right\|_{L^{2}(\mathbf{R})}^{2} \leq C\left\|e^{-y t} h(t)\right\|_{L^{2}[-\pi, \pi]}^{2} .
$$

Since the $y$ 's are being chosen from a compact set, there is a constant $C$ such that $\left\|e^{-y t} h(t)\right\|^{2} \leq C\|h(t)\|^{2}$. Applying Fubini's Theorem, we have

$$
\int_{y=1 / 2}^{1 / 2} \int_{x=-\infty}^{\infty} G(x+i y)^{2} d x d y \leq \int_{y=-1 / 2}^{1 / 2} C\|h\|_{L^{2}[-\pi, \pi]}^{2} d y \leq C\|h\|_{L^{2}[-\pi, \pi]}^{2}
$$


Remark. It follows from Lemma 2 that if $h(t)=\sum_{k=-\infty}^{\infty} a_{k} e^{i k t}$ and

$$
R^{*} h(j)=\sup _{\varepsilon>0}\left|\sum_{k=-\infty}^{\infty} \frac{e^{i(k+j) \varepsilon} a_{k+j}}{k}\right|,
$$

then $\left\|R^{*}(h)\right\|_{l^{2}(\mathrm{Z})} \leq C\|h\|_{L^{2}[-\pi, \pi]}$ (because $R^{*}$ differs from $I^{*}$ by an operator which is clearly strong $(2,2))$. This is not a robust enough result to survive transference to the ergodic context. We need instead to provide an estimate for

$$
a_{*}^{*}(j)=\sup _{n \geq 1} \sup _{\varepsilon>0}\left|\sum_{k=-n}^{n} \frac{e^{i(k+j) \varepsilon} a_{k+j}}{k}\right| ;
$$

this is the content of Lemma 3, which follows from Lemma 2 by means of the covering lemma and disjointification techniques also used in Petersen [1983]. We conjecture that even strong $(2,2)$ holds: $\left\|a_{*}^{*}\right\|_{l^{2}(\mathbf{Z})} \leq C\|a\|_{l^{2}(\mathbf{Z})}$.

Lemma 3. There is a constant $C>0$ such that if $\left\{a_{k}\right\} \in l^{2}(\mathbf{Z})$ and $\lambda>0$ then

$$
\operatorname{card}\left\{j: a_{*}^{*}(j)>\lambda\right\} \leq \frac{C}{\lambda^{2}} \sum_{k=-\infty}^{\infty}\left|a_{k}\right|^{2} .
$$

Proof. Let $A$ be a bounded subset of

$$
\left\{j: \sup _{n \geq 1} \sup _{\varepsilon>0}\left|\sum_{k=-n}^{n} \frac{e^{i(k+j) \varepsilon} a_{k+j}}{k}\right|>\lambda\right\},
$$

so that $A \subset[-N, N]$, say. For each $j \in A$ there is a block in $\mathbf{Z}$ of the form $\left[j-n_{j}, j+n_{j}\right]$ and an $\varepsilon_{j}>0$ such that

$$
\left|\sum_{k \in I_{j}} \frac{e^{i k \varepsilon_{j}} a_{k}}{k-j}\right|>\lambda
$$

Since

$$
\sum_{k \in I_{j}}^{\prime}=\sum_{k=-\infty}^{\infty}-\sum_{k \notin I_{j}},
$$

we may apply the triangle inequality to see that

$$
\begin{aligned}
A \subset & \left\{j: \sup _{\varepsilon>0}\left|\sum_{k=-\infty}^{\infty} \frac{e^{i k \varepsilon} a_{k}}{k-j}\right|>\frac{\lambda}{2}\right\} \\
& \cup\left\{j \in[-N, N]:\left|\sum_{k \notin I_{j}} \frac{e^{i k \varepsilon_{1}} a_{k}}{k-j}\right|>\frac{\lambda}{2}\right\}=A_{1} \cup A_{2} .
\end{aligned}
$$

If $j \in A_{1}$, then by Lemma 2 (since strong $(2,2)$ implies weak $(2,2)$ ), $j$ falls into a single (independent of $j$ ) set of cardinality no more than $\left(4 C / \lambda^{2}\right)\|a\|_{2}^{2}$. To count the $j$ 's in $A_{2}$, we first make each of the numerators real and nonnegative 
by taking the positive and negative parts of the real and imaginary parts. Thus we define $r_{j}^{+}(k)=\left[\operatorname{Re}\left(e^{i k \varepsilon_{j}} a_{k}\right)\right]^{+}$, and similarly define $r_{j}^{-}, i_{j}^{+}$, and $i_{j}^{-}$. We will count

$$
\left\{j \in A_{2}: \sum_{k \notin I_{j}} \frac{r_{j}^{+}(k)}{k-j}<-\frac{\lambda}{8}\right\}=r^{+}\left(A_{2}\right) .
$$

A similar argument will count how often the same sum is larger than $\lambda / 8$, and also how often the same sum with $r_{j}^{+}$replaced by $r_{j}^{-}, i_{j}^{+}$, and $i_{j}^{-}$is greater than $\lambda / 8$ and less than $-\lambda / 8$, giving the same estimate each time.

Replace the family $\left\{I_{j}\right\}$ by a disjoint subfamily $\left\{\tilde{I}_{j}\right\}$ which still covers at least $1 / 3$ of $A_{2}$. Index the centers of the new intervals by $\widetilde{J}$. If for $t \in \mathbf{R}$ we let

$$
h_{j}(t)=\sum_{k \notin \widetilde{I}_{j}} \frac{r_{j}^{+}(k)}{k-t},
$$

then $h_{j}^{\prime}(t)>0$. Since $h_{j}(j)<-\lambda / 8$, so is $h_{j}(n)<-\lambda / 8$ for $n \in\left[j-n_{j}, j\right]$. Hence we find

$$
\begin{aligned}
\operatorname{card} r^{+}\left(A_{2}\right) & \leq 6 \sum_{j \in \widetilde{J}}\left(n_{j}+1\right) \leq 6 \operatorname{card} \bigcup_{j \in \widetilde{J}}\left\{n: \sum_{k \notin \widetilde{I}_{j}} \frac{r_{j}^{+}(k)}{k-n}<-\frac{\lambda}{8}\right\} \\
\leq & 6 \operatorname{card}\left[\bigcup_{j \in \widetilde{J}}\left(\left\{n: \sum_{k=-\infty}^{\infty} \frac{r_{j}^{+}(k)}{k-n}<-\frac{\lambda}{16}\right\} \cup\left\{n: \sum_{k \in \widetilde{I}_{j}} \frac{r_{j}^{+}(k)}{k-n}>\frac{\lambda}{16}\right\}\right)\right] \\
\leq & \operatorname{card}\left[\bigcup_{j \in \widetilde{J}}\left\{n: \sum_{k=-\infty}^{\infty} \frac{r_{j}^{+}(k)}{k-n}<-\frac{\lambda}{16}\right\}\right]+6 \sum_{j \in \widetilde{J}}\left(\frac{(16)^{2} C}{\lambda^{2}} \sum_{k \in \widetilde{I}_{j}}\left|a_{k}\right|^{2}\right),
\end{aligned}
$$

by Lemma 2 . Continuing, because the $\tilde{I}_{j}$ are disjoint all of this is

$$
\begin{aligned}
& \leq 6 \text { card }\left\{n: \sup _{\varepsilon>0}\left|\sum_{k=-\infty}^{\infty} \frac{e^{i k \varepsilon} a_{k}}{k-n}\right|>\frac{\lambda}{16}\right\}+\frac{6(16)^{2} C}{\lambda^{2}} \sum_{k=-\infty}^{\infty}\left|a_{k}\right|^{2} \\
& \leq \frac{6(16)^{2} C}{\lambda^{2}} \sum_{k=-\infty}^{\infty}\left|a_{k}\right|^{2}+\frac{6(16)^{2} C}{\lambda^{2}} \sum_{k=-\infty}^{\infty}\left|a_{k}\right|^{2},
\end{aligned}
$$

where we have again applied Lemma 2 , as in the remark above. The estimate on $r^{+}\left(A_{2}\right)$ is now complete with constant equal to $(12)(16)^{2} C$, where $C$ is the constant from Lemma 2 . We may similarly estimate $r^{-}\left(A_{2}\right), i^{+}\left(A_{2}\right)$, and $i^{-}\left(A_{2}\right)$, where the definitions of these sets are obvious. Combining these estimates on $A_{2}$ with the previously obtained estimate on $A_{1}$, we obtain the estimate in the conclusion of Lemma 3 , where the constant for the estimate in line (22) may be taken as $(2)(4)(12)(16)^{2} C$. 
Proof of Lemma 1. For each fixed $N \geq 1$ define

$$
A_{N}=\left\{x: \sup _{\varepsilon>0} \sup _{1 \leq n \leq N}\left|\frac{1}{\pi} \sum_{k=-n}^{n} \frac{e^{i k \varepsilon} f\left(T^{k} x\right)}{k}\right|>\lambda\right\},
$$

and for this $N$ and $K \in \mathbf{N}$ define

$$
\bar{A}_{N, K}=\left\{(x, j):-K \leq j \leq K \text { and } T^{j} x \in A_{N}\right\} .
$$

An argument analogous to that given in Petersen [1983] to prove Lemma 1 of that paper shows that

$$
\mu\left(A_{N}\right)=\frac{1}{2 K+1} \mu x \operatorname{card}\left(\bar{A}_{N, K}\right) \leq\left(\frac{C}{\lambda^{2}}\right) \frac{[2(K+N)+1]}{2 K+1}\|f\|_{2}^{2},
$$

where card denotes counting measure on $Z$. Lemma 1 follows by letting $K$ tend to infinity.

To complete the proof of Theorem 2, fix any nonnegative sequence $\left\{\varepsilon_{k}\right\}$ tending to 0 . We have shown that the a.e. convergence claimed in the conclusion of Theorem 2 holds for a dense set of functions in $L^{2}$. The maximal inequality provided by Lemma 1 implies, by Banach's Principle, that the set of functions in $L^{2}$ for which this same a.e. convergence holds must be closed. Hence it must be all of $L^{2}$.

Remark. Probably a Wiener-Wintner type theorem holds in this situation; that is, for each $f \in L^{2}$ there probably exists a single set of measure 0 outside of which $H_{\varepsilon} f(x)$ exists for all $\varepsilon$. Then these arguments would extend to show that in fact

$$
\lim _{\varepsilon \rightarrow 0^{+}} H_{\varepsilon} f(x)=H f(x)+i E\{0\} f(x) \text { a.e. for each } f \in L^{2}
$$

\section{REFERENCES}

J. T. Campbell, [1986] Spectral analysis of the ergodic Hilbert transform, Indiana Univ. Math. J. 35, 379-390.

M. Cotlar, [1955] A unified theory of Hilbert transforms and ergodic theorems, Rev. Mat. Cuyana 1, 105-167.

R. Duncan, [1977] Some pointwise convergence results in $L^{p}(\mu), 1<p<\infty$, Canad. Math. Bull. 20, 277-284.

V. F. Gaposhkin, [1981] Individual ergodic theorem for normal operators in $L^{2}$, Functional Anal. Appl. 15, 18-22.

L. Hörmander, [1973] An introduction to complex analysis in several variables, North-Holland, Amsterdam,

C. Kenig and P. Tomas, [1980] Maximal operators defined by Fourier multipliers, Studia Math. 68, 79-83.

U. Krengel, [1985] Ergodic theorems, De Gruyter, Berlin and New York. 
K. Petersen, [1983] Another proof of the existence of the ergodic Hilbert transform, Proc. Amer. Math. Soc. 88, 39-44.

N. Wiener and A. Wintner, [1941] Harmonic analysis and ergodic theory, Amer. J. Math. 63, 415426.

Department of Mathematical Sciences, Memphis State University, Memphis, TennesSEE 38152

Department of Mathematics, University of North Carolina, Chapel Hill, North CarOLINA 27599 